\title{
SUBMANIFOLDS WITH CONSTANT SCALAR CURVATURE
}

\author{
JINTANG Li
}

\begin{abstract}
Let $M^{n}$ be a compact submanifold of $S^{n+p}(c)$ with constant scalar curvature. In this paper, we prove that if the squared norm $S$ of the second fundamental form satisfies a certain inequality, then $M^{n}$ is a totally umbilic or eqality holds and we described all $M^{n}$ that satisfy this equality.
\end{abstract}

\section{Introduction}

Let $S^{n+p}(c)$ be a sphere of constant sectional curvature $c$ and let $M^{n}$ be a compact submanifold of $S^{n+p}(c)$ with constant scalar curvature. Let $S=$ $\sum_{i, j, \alpha}\left(h_{i j}^{\alpha}\right)^{2}$ and $\xi$ be the square norm of the second fundamental form and the mean curvature vector of $M$ respectively. We set $H=\|\xi\|, S_{\alpha}=\sum_{i, j}\left(h_{i j}^{\alpha}\right)^{2}$ and $H^{\alpha}=\left(h_{i j}^{\alpha}\right)_{n \times n}$. In [1], Li Haizhong studied some properties for $p=1$. The purpose of this paper is to extend the result of [1] to higher codimensions. In other words, we shall prove the following:

Theorem 1. Let $M^{n}(2 \leq n \leq 4$, when $n=2, p \neq 1,2)$ be a compact submanifold of $S^{n+p}(c)$ with constant scalar curvature $\rho$. Assume the normalized mean curvature vector is parallel with respect to the normal connection. If

$$
\begin{aligned}
S-n \bar{R} \leq & \frac{B_{p}}{n-1-B_{p}}\left\{n^{2} c+n^{2} \bar{R}\right. \\
& \left.-\frac{n-2}{\sqrt{n-1}} \sqrt{[S+n(n-1) \bar{R}]\left[n S_{n-1}-S-n(n-1) \bar{R}\right]}\right\},
\end{aligned}
$$

where

$$
B_{p}= \begin{cases}1, & p=1,2 \\ \frac{2}{3}, & p \geq 3\end{cases}
$$

and $\bar{R}=\rho / n(n-1)-c$, then either

Mathematics Subject Classification (2000): $53 \mathrm{c} 42$.

Received July 29, 2003; revised April 21, 2004. 
or

(1) $S=n H^{2}$ and $M$ is totally umbilic.

(2) The equality holds in (1.1), $H$ is a constant and one of the following cases occurs:

(a) $H=0, p=1$ and $M$ is a minimal Clifford hypersurface,

$$
M^{n}=S^{m}\left(\left(\frac{m}{n c}\right)^{1 / 2}\right) \times S^{n-m}\left(\left(\frac{n-m}{n c}\right)^{1 / 2}\right) \hookrightarrow S^{n+p}(c) .
$$

(b) $H \neq 0, \quad p=1$ and $M^{n}=S^{n-1}\left(r_{1}\right) \times S^{1}\left(r_{2}\right)$, where $r_{1}^{2}+r_{2}^{2}=c^{-1}$, $r_{1}^{2}<(n-1) / n c$.

(c) $H \neq 0, p=2$ and $M$ is a minimal Clifford hypersurface in a hypersphere, $M^{n}=S^{m}\left(\left(\frac{m}{n\left(c+H^{2}\right)}\right)^{1 / 2}\right) \times S^{n-m}\left(\left(\frac{n-m}{n\left(c+H^{2}\right)}\right)^{1 / 2}\right) \subset S^{n+1}\left(c+H^{2}\right) \hookrightarrow S^{n+2}(c)$.

(d) $H \neq 0, p=2$ and for all $0<H_{2} \leq H, M$ is an $H_{1}$-torus,

$$
M^{n}=S^{n-1}\left(r_{1}\right) \times S^{1}\left(r_{2}\right) \subset S^{n+1}\left(c+H_{2}^{2}\right) \hookrightarrow S^{n+2}(c),
$$

where $H_{1}^{2}+H_{2}^{2}=H^{2}, r-1^{2}+r_{2}^{2}=\left(c+H_{2}^{2}\right)^{-1}, r_{1}^{2}<(n-1) / n\left(c+H_{1}^{2}\right)$, and an $H_{1}$-torus is defined by Walcy Santos (see [3]).

(e) $H \neq 0, n=2, p=3$ and $M^{2}$ is a Veronese surface in a hypersphere,

$$
M^{2} \subset S^{4}\left(c+H^{2}\right) \hookrightarrow S^{5}(c) .
$$

for $n \geq 5$, we have

THEOREM 2. Let $M^{n}(n \geq 5)$ be a compact submanifold of $S^{n+p}(c)$ with constant scalar curvature $\rho$. Assume the normalized mean curvature vector is parallel with respect to the normal connection and $\rho \geq n(n-1) c$. If $S$ and $H$ satisfied (1.1), then either

(1) $S=n H^{2}$ and $M$ is totally umbilic.

or

(2) The equality holds in (1.1), $H$ is a constant and one of the following cases occurs:

(a) $H \neq 0, \quad p=1$ and $M^{n}=S^{n-1}\left(r_{1}\right) \times S^{1}\left(r_{2}\right)$, where $r_{1}^{2}+r_{2}^{2}=c^{-1}$, $r_{1}^{2}<(n-1) / n c$.

(b) $H \neq 0, p=2$ and $M$ is a minimal Clifford hypersurface in a hypersphere, $M^{n}=S^{m}\left(\left(\frac{m}{n\left(c+H^{2}\right)}\right)^{1 / 2}\right) \times S^{n-m}\left(\left(\frac{n-m}{n\left(c+H^{2}\right)}\right)^{1 / 2}\right) \subset S^{n+1}\left(c+H^{2}\right) \hookrightarrow S^{n+2}(c)$.

(c) $H \neq 0, p=2$ and for all $0<H_{2} \leq H, M$ is an $H_{1}$-torus,

$$
M^{n}=S^{n-1}\left(r_{1}\right) \times S^{1}\left(r_{2}\right) \subset S^{n+1}\left(c+H_{2}^{2}\right) \hookrightarrow S^{n+2}(c),
$$

where $H_{1}^{2}+H_{2}^{2}=H^{2}, r-1^{2}+r_{2}^{2}=\left(c+H_{2}^{2}\right)^{-1}, r_{1}^{2}<(n-1) / n\left(c+H_{1}^{2}\right)$. 
Remark 1. When $p=1, n=3,4$, our assumption condition (1.1), i.e.

$$
S-n \bar{R} \leq \frac{1}{n-2}\left\{n^{2} c+n^{2} \bar{R}-(n-2) \sqrt{[S+n(n-1) \bar{R}][S-n \bar{R}]}\right\}
$$

is equivalent to

$$
(n-2)^{2}[S+n(n-1) \bar{R}][S-n \bar{R}] \leq\left\{n^{2} c+2 n(n-1) \bar{R}-(n-2) S\right\}^{2} .
$$

On the other hand, $\left(1.1^{\prime}\right)$ is equivalent to

$$
S \leq \frac{n}{(n-2)(n \bar{R}+2)}\left[n(n-1) \bar{R}^{2}+4(n-1) \bar{R}+n\right],
$$

so Theorem 1 improve Theorem 2 of [1].

Remark 2. In Theorem 2, when the equality holds in (1.1), we have that $H \neq 0$ by $\rho \geq n(n-1) c$ and the Guass equation.

\section{Priliminaries}

We choose local field of orthonormal frame $\left\{e_{1}, \ldots, e_{n+p}\right\}$ in such way that $e_{n+1}$ is the normalized mean curvature vector and $h_{i j}^{n+1}=\lambda_{i} \delta_{i j}$. We need the following Lemma:

LEMma 1. If $2 \leq n \leq 4$, then

$$
\int_{M} \sum_{i, j, k, \alpha}\left\{\left(h_{i j k}^{\alpha}\right)^{2}+h_{i j}^{\alpha} h_{k k i j}^{\alpha}\right\} \geq 0 .
$$

Proof.

$$
\begin{aligned}
& \sum_{i, j, k, \alpha} h_{i j}^{\alpha} h_{k k i j}^{\alpha}=\sum_{j} \nabla_{e_{j}}\left(\sum_{i, k, \alpha} h_{i j}^{\alpha} h_{k k i}^{\alpha}\right)-\sum_{i, j, k, \alpha} h_{i j j}^{\alpha} h_{k k i}^{\alpha} \\
&=\sum_{j} \nabla_{e_{j}}\left(\sum_{i, k, \alpha} h_{i j}^{\alpha} h_{k k i}^{\alpha}\right)-\sum_{i, \alpha}\left(\sum_{j} h_{j j i}^{\alpha}\right)^{2} \\
&=\sum_{j} \nabla_{e_{j}}\left(\sum_{i, k, \alpha} h_{i j}^{\alpha} h_{k k i}^{\alpha}\right)-\sum_{i, \alpha}\left(h_{i i i}^{\alpha}\right)^{2}-\sum_{i, \alpha}\left(\sum_{j \neq i} h_{j j i}^{\alpha}\right)^{2}, \\
& \sum_{i, j, k, \alpha}\left(h_{i j k}^{\alpha}\right)^{2}=\sum_{i, \alpha}\left(h_{i i i}^{\alpha}\right)^{2}+3 \sum_{i \neq j, \alpha}\left(h_{j j i}^{\alpha}\right)^{2}+\sum_{i, j, k \neq \alpha}\left(h_{i j k}^{\alpha}\right)^{2} .
\end{aligned}
$$

For $n \leq 4$ and $\forall i$, we have 


$$
3 \sum_{j \neq i}\left(h_{j j i}^{\alpha}\right)^{2} \geq(n-1) \sum_{j \neq i}\left(h_{j j i}^{\alpha}\right)^{2} \geq\left(\sum_{j \neq i} h_{j j i}^{\alpha}\right)^{2},
$$

so we have

$$
3 \sum_{j \neq i, \alpha}\left(h_{j j i}^{\alpha}\right)^{2} \geq \sum_{i, \alpha}\left(\sum_{j \neq i} h_{j j i}^{\alpha}\right)^{2} .
$$

By (2.1), (2.2), (2.3) and the compactness of $M$, the Lemma 1 is true. then

Lemma 2 [1]. Assume the scalar curvature $\rho=$ constant and $\rho \geq n(n-1) c$,

$$
\sum_{i, j, k, \alpha}\left\{\left(h_{i j k}^{\alpha}\right)^{2}-\sum_{i}\left(n H_{i}\right)^{2}\right\} \geq 0 .
$$

Lemma 3 [2]. $\quad M^{n}$ is a compact submanifold of $S^{n+p}(c)$ with parallel normalized mean curvature vector if and only if $H^{n+1} H^{\alpha}=H^{\alpha} H^{n+1}$.

Lemma 4 [3]. Let $A, B: R^{n} \rightarrow R^{n}$ be symmetric linear maps such that $[A, B]=0$ and $\operatorname{tr} A=\operatorname{tr} B=0$, then

$$
-\frac{n-2}{\sqrt{n(n-1)}}\left(\operatorname{tr} A^{2}\right)\left(\operatorname{tr} B^{2}\right)^{1 / 2} \leq \operatorname{tr} A^{2} B \leq \frac{n-2}{\sqrt{n(n-1)}}\left(\operatorname{tr} A^{2}\right)\left(\operatorname{tr} B^{2}\right)^{1 / 2},
$$

and the equality holds on the right hand (resp. the left hand) side if and only if $n-1$ of the eigenvalues $x_{i}$ of $A$ and the corresponding eigenvalues $y_{i}$ of $B$ satisfy

$$
\begin{gathered}
\left|x_{i}\right|=\frac{\left(\operatorname{tr} A^{2}\right)^{1 / 2}}{\sqrt{n(n-1)}}, \quad x_{i} x_{j} \geq o, \\
y_{i}=\frac{\left(\operatorname{tr} B^{2}\right)^{1 / 2}}{\sqrt{n(n-1)}} \cdot \quad\left(\operatorname{resp} . \quad y_{i}=-\frac{\left(\operatorname{tr} B^{2}\right)^{1 / 2}}{\sqrt{n(n-1)}}\right)
\end{gathered}
$$

Lemma 5 [4]. Let $A_{n+1}, \ldots, A_{n+p}$ be symmetric $(n \times n)$-matrices. Denote $S_{\alpha \beta}=\operatorname{tr}\left(A_{\alpha} A_{\beta}\right), S_{\alpha}=S_{\alpha \alpha}=N\left(A_{\alpha}\right), S=\sum_{\alpha} S_{\alpha}$, then

$$
\sum_{\alpha, \beta} N\left(A_{\alpha} A_{\beta}-A_{\beta} A_{\alpha}\right)+\sum_{\alpha, \beta} S_{\alpha \beta}^{2} \leq\left(1+\frac{1}{2} \operatorname{sgn}(p-1)\right) S^{2} .
$$

\section{The of Theorem}

We define a self-adjoint operator $\square$ by [1]

$$
\square f=\sum_{i, j}\left(n H \delta_{i j}-h_{i j}^{n+1}\right) f_{i j} .
$$


we have [2]

$$
\begin{aligned}
\frac{1}{2} \triangle S= & \sum_{i, j, k, \alpha}\left(h_{i j k}^{\alpha}\right)^{2}+\sum_{i, j, k, \alpha} h_{i j}^{\alpha} h_{k k i j}^{\alpha}+2 \sum_{\alpha, \beta} \operatorname{tr}\left(H^{\alpha} H^{\beta}\right)^{2}-2 \sum_{\alpha, \beta} \operatorname{tr}\left(H^{\alpha}\right)^{2}\left(H^{\beta}\right)^{2} \\
& -\sum_{\alpha, \beta}\left[\operatorname{tr}\left(H^{\alpha} H^{\beta}\right)\right]^{2}+\sum_{\alpha, \beta} \operatorname{tr} H^{\beta}\left[\operatorname{tr}\left(H^{\alpha}\right)^{2} H^{\beta}\right]+c n S-c n^{2} H^{2} .
\end{aligned}
$$

By the Guass equation and the scalar curvature $\rho=$ constant, we get

$$
\begin{aligned}
\square(n H)= & \frac{1}{2} \triangle(n H)^{2}-\sum_{i}\left(n H_{i}\right)^{2}-\sum_{i}(n H)_{i i} \lambda_{i} \\
= & \frac{1}{2} \triangle S-\sum_{i}\left(n H_{i}\right)^{2}-\sum_{i}(n H)_{i i} \lambda_{i} \\
= & \sum_{i, j, k, \alpha}\left(h_{i j k}^{\alpha}\right)^{2}-\sum_{i}\left(n H_{i}\right)^{2}+2 \sum_{\alpha, \beta} \operatorname{tr}\left(H^{\alpha} H^{\beta}\right)^{2}-2 \sum_{\alpha, \beta} \operatorname{tr}\left(H^{\alpha}\right)^{2}\left(H^{\beta}\right)^{2} \\
& -\sum_{\alpha, \beta}\left[\operatorname{tr}\left(H^{\alpha} H^{\beta}\right)\right]^{2}+\sum_{\alpha, \beta} \operatorname{tr} H^{\beta}\left[\operatorname{tr}\left(H^{\alpha}\right)^{2} H^{\beta}\right]+c n S-c n^{2} H^{2} .
\end{aligned}
$$

From Lemma 3 and Lemma 5, we have, $p \geq 2$,

$$
\begin{aligned}
2 \sum_{\alpha, \beta} \operatorname{tr}( & \left.H^{\alpha} H^{\beta}\right)^{2}-2 \sum_{\alpha, \beta} \operatorname{tr}\left(H^{\alpha}\right)^{2}\left(H^{\beta}\right)^{2}-\sum_{\alpha, \beta}\left[\operatorname{tr}\left(H^{\alpha} H^{\beta}\right)\right]^{2} \\
= & 2 \sum_{\alpha \neq \beta \neq n+1} \operatorname{tr}\left(H^{\alpha} H^{\beta}\right)^{2}-2 \sum_{\alpha \neq \beta \neq n+1} \operatorname{tr}\left(H^{\alpha}\right)^{2}\left(H^{\beta}\right)^{2} \\
& -\sum_{\alpha \neq n+1, \beta \neq n+1}\left[\operatorname{tr}\left(H^{\alpha} H^{\beta}\right)\right]^{2}-S_{n+1}^{2}-2 \sum_{\alpha \neq n+1}\left[\operatorname{tr}\left(H^{\alpha} H^{n+1}\right)\right]^{2} \\
= & -N_{\alpha \neq \beta \neq n+1}\left(H^{\alpha} H^{\beta}-H^{\beta} H^{\alpha}\right)-\sum_{\alpha \neq n+1, \beta \neq n+1} S_{\alpha \beta}^{2} \\
& -S_{n+1}^{2}-2 \sum_{\alpha \neq n+1}\left[\operatorname{tr}\left(H^{\alpha} H^{\beta}\right)\right]^{2} \\
\geq & -\left(1+\frac{1}{2} \operatorname{sgn}(p-2)\right)\left(\sum_{\alpha \neq n+1} S_{\alpha}\right)^{2}-S_{n+1}^{2}-2 \sum_{\alpha \neq n+1}\left[\sum_{i, j}\left(h_{i j}^{n+1}-H \delta_{i j}\right) h_{i j}^{\alpha}\right]^{2} \\
\geq & -\frac{1}{B_{p}}\left(S-S_{n+1}\right)^{2}-S_{n+1}^{2}-2 \sum_{i, j}\left(h_{i j}^{n+1}-H \delta_{i j}\right)^{2} \sum_{i, j, \alpha \neq n+1}\left(h_{i j}^{\alpha}\right)^{2} \\
\geq & -\frac{1}{B_{p}}\left(S-S_{n+1}\right)^{2}-S_{n+1}^{2}-2\left(S_{n+1}-n H^{2}\right)\left(S-S_{n+1}\right) .
\end{aligned}
$$

Obviously, the inequality (3.3) holds for $p=1$. 
By Lemma 4, we take

$$
\begin{aligned}
\sum_{\alpha, \beta} \operatorname{tr} & H^{\beta} \operatorname{tr}\left[\left(H^{\alpha}\right)^{2} H^{\beta}\right] \\
= & n H \sum_{i} \lambda_{i}^{3}+n H \sum_{\alpha \neq n+1} \operatorname{tr}\left[\left(H^{\alpha}\right)^{2} H^{n+1}\right] \\
= & 3 n H^{2} S_{n+1}-2 n^{2} H^{4}-n H \sum_{i}\left(H-\lambda_{i}\right)^{3} \\
& +n H \sum_{\alpha \neq n+1} \operatorname{tr}\left[\left(H^{\alpha}\right)^{2}\left(H^{n+1}-H I_{n \times n}\right)\right]+n H \sum_{\alpha \neq n+1} \operatorname{tr}\left[\left(H^{\alpha}\right)^{2} H I_{n \times n}\right] \\
\geq & 3 n H^{2} S_{n+1}-2 n^{2} H^{4}-\frac{n(n-2)}{\sqrt{n(n-1)}} H\left(S_{n+1} n H^{2}\right)^{3 / 2} \\
& -\frac{n(n-2)}{\sqrt{n(n-1)}} H\left(S-S_{n+1}\right)\left(S_{n+1}-n H^{2}\right)^{1 / 2}+n H^{2}\left(S-S_{n+1}\right) \\
= & 2 n H^{2} S_{n+1}-2 n^{2} H^{4}+n H^{2} S-\frac{n(n-2)}{\sqrt{n(n-1)}} H\left(S-n H^{2}\right)\left(S_{n+1}-n H^{2}\right)^{1 / 2},
\end{aligned}
$$

and the equation holds in (3.4) if and only if

$$
H-\lambda_{i}=-\frac{S^{1 / 2}}{\sqrt{n(n-1)}}, \quad(i=1, \ldots, n-1) .
$$

It follows from (3.1), (3.2), (3.3) and (3.4) that

$$
\begin{aligned}
\frac{1}{2} \triangle S \geq & \sum_{i, j, k, \alpha}\left(h_{i j k}^{\alpha}\right)^{2}+\sum_{i, j, k, \alpha} h_{i j}^{\alpha} h_{k k i j}^{\alpha}-\frac{1}{B_{p}} S^{2}-\left(\frac{1}{B_{p}}-1\right) S_{n+1}^{2} \\
& +2\left(\frac{1}{B_{p}}-1\right) S S_{n+1}+3 n H^{2} S-2 n^{2} H^{4} \\
& -\frac{n(n-2)}{\sqrt{n(n-1)}} H\left(S-n H^{2}\right)\left(S_{n+1}-n H^{2}\right)^{1 / 2}+n c\left(S-n H^{2}\right) \\
= & \sum_{i, j, k, \alpha}\left(h_{i j k}^{\alpha}\right)^{2}+\sum_{i, j, k, \alpha} h_{i j}^{\alpha} h_{k k i j}^{\alpha} \\
& +\left(\frac{1}{B_{p}}-1\right)\left(S_{n+1}-n H^{2}\right)\left(2 S-S_{n+1}-n H^{2}\right) \\
& -\frac{1}{B_{p}}\left(S-n H^{2}\right)^{2}+\left(n H^{2}+n c\right)\left(S-n H^{2}\right) \\
& -\frac{n(n-2)}{\sqrt{n(n-1)}} H\left(S-n H^{2}\right)\left(S_{n+1}-n H^{2}\right)^{1 / 2}
\end{aligned}
$$


and

$$
\begin{aligned}
\geq \sum_{i, j, k, \alpha}\left(h_{i j k}^{\alpha}\right)^{2}+ & \sum_{i, j, k, \alpha} h_{i j}^{\alpha} h_{k k i j}^{\alpha} \\
+\left(S-n H^{2}\right)\{ & -\frac{1}{B_{p}}\left(S-n H^{2}\right)+n H^{2}+n c \\
& \left.\quad-\frac{n(n-2)}{\sqrt{n(n-1)}} H\left(S_{n+1}-n H^{2}\right)^{1 / 2}\right\},
\end{aligned}
$$

$$
\begin{aligned}
\square(n H) \geq & \sum_{i, j, k, \alpha}\left(h_{i j k}^{\alpha}\right)^{2}-\sum_{i}\left(n H_{i}\right)^{2} \\
+\left(S-n H^{2}\right)\{ & -\frac{1}{B_{p}}\left(S-n H^{2}\right)+n H^{2}+n c \\
& \left.\quad-\frac{n(n-2)}{\sqrt{n(n-1)}} H\left(S_{n+1}-n H^{2}\right)^{1 / 2}\right\} .
\end{aligned}
$$

When $2 \leq n \leq 4$, it follows from (3.6) and Lemma 1 that

$$
\begin{aligned}
\int_{M} \frac{1}{2} \triangle S d v \geq \int_{M}\left(S-n H^{2}\right)\{ & -\frac{1}{B_{p}}\left(S-n H^{2}\right)+n H^{2}+n c \\
& \left.-\frac{n(n-2)}{\sqrt{n(n-1)}} H\left(S_{n+1}-n H^{2}\right)^{1 / 2}\right\} d v .
\end{aligned}
$$

When $n \geq 5$, it follows from (3.7) and Lemma 2 that

$$
\begin{aligned}
\square(n H) \geq\left(S-n H^{2}\right)\{ & -\frac{1}{B_{p}}\left(S-n H^{2}\right)+n H^{2}+n c \\
& \left.-\frac{n(n-2)}{\sqrt{n(n-1)}} H\left(S_{n+1}-n H^{2}\right)^{1 / 2}\right\} .
\end{aligned}
$$

By $\bar{R}=(\rho-n(n-1) c) / n(n-1)=\left(n^{2} H^{2}-S\right) / n(n-1)$, it can be seen that our assumption condition (1.1), i.e.

$$
S-n \bar{R} \leq \frac{B_{p}}{n-1-B_{p}}\left\{n^{2} c+n^{2} \bar{R}\right.
$$

is equivalent to

$$
\left.-\frac{n-2}{\sqrt{n-1}} \sqrt{[S+n(n-1) \bar{R}]\left[n S_{n-1}-S-n(n-1) \bar{R}\right]}\right\}
$$

$$
\begin{aligned}
\frac{n S-n^{2} H^{2}}{n-1} \leq \frac{B_{p}}{n-1-B_{p}}\{ & n^{2} c+n^{2} H^{2}-\frac{n}{n-1}\left(S-n H^{2}\right) \\
& \left.-\frac{n^{2}(n-2)}{\sqrt{n(n-1)}} H\left(S_{n+1}-n H^{2}\right)^{1 / 2}\right\},
\end{aligned}
$$


so it is also equivalent to

$$
-\frac{1}{B_{p}}\left(S-n H^{2}\right)+n H^{2}+n c-\frac{n(n-2)}{\sqrt{n(n-1)}} H\left(S_{n+1}-n H^{2}\right)^{1 / 2} \geq 0,
$$

therefore the right hand side of (3.8), (3.9) is non-negative. Since $M$ is compact, we have that either $S=n H^{2}, M$ is a totally umbilic or

$$
\begin{aligned}
S-n \bar{R}= & \frac{B_{p}}{n-1-B_{p}}\left\{n^{2} c+n^{2} \bar{R}\right. \\
& \left.-\frac{n-2}{\sqrt{n-1}} \sqrt{[S+n(n-1) \bar{R}]\left[n S_{n-1}-S-n(n-1) \bar{R}\right]}\right\} .
\end{aligned}
$$

In the latter case, by (3.5), we have

$$
S_{n+1}=(n-1)\left(H+\frac{S^{1 / 2}}{\sqrt{n(n-1)}}\right)^{2}+\left(H-\sqrt{\frac{(n-1) S}{n}}\right)^{2} .
$$

By the Guass equation, we get

$$
S=n^{2} H^{2}+n(n-1) c-\rho .
$$

By (3.10), (3.11), (3.12) and $\bar{R}=\rho / n(n-1)-c=$ constant, we take that $H$ is a constant, thus we complete the proof of Theorem 1, 2 by Theorem 1.6 of [3].

Acknowledgement. The author wishes to thank the referee for his close reading of the first draft of this paper which led to various improvements.

\section{REFERENCES}

[ 1 ] Li Haizhong, Hypersurface with Constant Scalar Curvature in Spaceforms, Math. Ann., 305 (1996), 665-675.

[2] S. T. Yan, Submanifolds with Constant Mean Curvature, Amer. J. Math., 96 (1974), 346466, 97 (1975), 76-100.

[3] Walcy Santos, Submanifolds with Parallel Mean Curvature Vector in Spheres, Tohoku Math. J. 46 (1994), 403-415.

[4] A. M. Li AND J. M. Li, An Intrinsic Rigidity Theorem for Minimal Submanifolds in a sphere, Arch. Math. 58 (1992), 582-594.

[ 5 ] S. Y. Cheng And S. T. Yan, Hypersurfaces with Constant Scalar Curvature, Math. Ann. 225 (1977), 195-204.

Department of Mathematics

Xiammen University, 361005 Xiammen, Fujian

P.R. CHINA 